\title{
Synthesis and characterization of thermo-sensitive magnetic maghemite nanoparticles
}

\author{
Ariel L. Cappelletti, Julieta I. Paez and Miriam C. Strumia* \\ Departamento de Química Orgánica (IMBIV-CONICET) Facultad de Ciencias Químicas, \\ Universidad Nacional de Córdoba, Medina Allende y Haya de la Torre, Ciudad Universitaria, \\ Córdoba (5000), Argentina \\ E-mail: mcs@fcq.unc.edu.ar
}

\begin{abstract}
The modification of magnetic nanoparticles (MNPs) with a thermo-sensitive organic shell of poly( $N$-isopropylacrylamide) (PNIPAm) is reported. The surface of maghemite MNPs was modified in two steps: firstly, using a silane coupling agent and leaving vinyl pending groups on the MNPs surface; and secondly, grafting PNIPAm chains from the modified MNPs via a free radical polymerization. The results indicate that the method developed here for the modification of MNPs is an effective way of synthesizing thermo-responsive MNPs. They present a magnetic behavior due to the maghemite core and a thermal response based on a deswelling/swelling of their polymeric shell (PNIPAm).
\end{abstract}

Keywords: Magnetic nanoparticles, maghemite, thermo-sensitive nanoparticles, poly $(N-$ isopropylacrylamide)

\section{Introduction}

The preparation of non-toxic nanoparticles exhibiting colloidal stability in biological media and offering anchoring sites is currently a real challenge. Iron oxide nanoparticles are by far the most used magnetic nanoparticles (MNPs) due to the following reasons: they are much less toxic than their metallic counterparts, and present high saturation magnetization and superparamagnetic behavior. Besides, MNPs generate heat via magnetic hysteresis loss, Neel-relaxation, and Brown-relaxation when exposed to an alternating magnetic field. Among MNPs, maghemite $(\gamma-$ $\left.\mathrm{Fe}_{2} \mathrm{O}_{3}\right)$ and magnetite $\left(\mathrm{Fe}_{3} \mathrm{O}_{4}\right)$ nanoparticles are excellent choices due to their already proven biocompatibility. By application of an external magnetic field, magnetic particles can be retained within a target organ for a given period of time, thus limiting spreading of the particles in the general circulation. ${ }^{1}$ 
On the other hand, recently the interest in thermo-sensitive polymers has increased exponentially due to their enormous potential in biotechnology and medicine. Thermallyresponsive polymers can collapse or expand on heating. This process exhibits swelling and deswelling structures respectively, above and below LCST. ${ }^{2}$ For example, microgels based on poly( $N$-isopropyl acrylamide) (PNIPAm), are really promising materials for biomedical applications since they exhibit a $\mathrm{LCST}^{3,4}$ at around $32{ }^{\circ} \mathrm{C}$. Besides, they are biocompatible, ${ }^{5}$ and PNIPAm hydrogels have commonly been referred to as smart materials because they can respond to an external stimulus (temperature) with an on-off switch mechanism. Hence, they are useful for drug delivery applications, e.g. for producing local hyperthermia in a tumor tissue, and delivering drug on-demand at local site of action.

Moreover, the combination of MNPs and thermally-responsive polymers (Figure 1) represents an important class of composite responsive material with potential applications in biomedical fields, such as magnetic resonance imaging (MRI), hyperthermia, bioassays, ${ }^{6}$ and drug or biomolecule transport. ${ }^{7,8}$

Some interesting research works on novel procedures of synthesis, chemical modification and applications of thermally-responsive MNPs have recently been published. Park ${ }^{9}$ has developed a novel route for the synthesis of the thermo-responsive core-shell MNPs through the covalent binding of vinyl carboxylic acid- $\beta$-cyclodextrins and further polymerization of NIPAm monomers. $\mathrm{Zhu}^{10}$ have reported the successful preparation of a novel magnetic targeting and temperature-responsive drug delivery system based on PNIPAm modified mesoporous silica SBA-15 containing maghemite MNPs. Wang ${ }^{11}$ have reported the preparation of antifouling thermo-sensitive MNPs for biomedical applications via surface free radical polymerization using poly(ethylene glycol)-monomethacrylate and $N$-isopropylacrylamide.

In this work, we report the preparation and characterization of thermo-sensitive magnetic maghemite nanoparticles. A two-step synthetic method was performed: firstly, MNPs were coated with 3-(methacriloxy)propyltrimethoxysilane (MEMO); secondly, PNIPAm chains were grafted from the modified MNPs via free radical polymerization. The obtained products were characterized by means of Infrared Spectroscopy (FT-IR), Thermogravimetric Analysis (TGA), UV-vis spectroscopy, Dynamic Light Scattering (DLS), Powder X-ray Diffraction (PXRD) and Transmission Electron Microscopy (TEM).

The results found indicated that the methodology here developed for the modification of MNPs is an effective way of synthesizing thermo-responsive MNPs. They present a magnetic behavior due to the maghemite core and a thermal response based on a deswelling/swelling of their polymeric shell (PNIPAm).

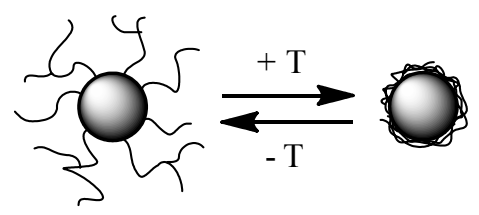

Figure 1. Thermo-responsive behavior of MNPs due to a temperature stimulus. 


\section{Results and Discussion}

\section{Synthesis of PNIPAm modified MNPs}

The preparation of thermo-sensitive MNPs was achieved following a "grafting from" method through radical polymerization, as depicted in Figure 2. The incorporation of a thermo-sensitive organic shell of PNIPAm onto the MNP surface was carried out in two synthetic steps.

Firstly, the MNPs were modified with the silane coupling agent MEMO. It leads onto the MNP surface a reactive double carbon bond able to react with vinyl monomers such as NIPAm. In addition, MEMO is expected to act as a spacer between MNPs and the grafted polymer chains. For the surface chemical functionalization different molar ratios of MEMO/MNPs were used, observing that the highest incorporation of MEMO was found at the molar ratio of MEMO/MNPs of 5:1 as proven by FT-IR. According to Schmidt, ${ }^{12}$ after reaction of MNPs with MEMO, self-condensation of silane groups is promoted by water removal, forming a polysiloxane film on the surface of the nanoparticle. Thus, azeotropic distillation under reduced pressure was performed.

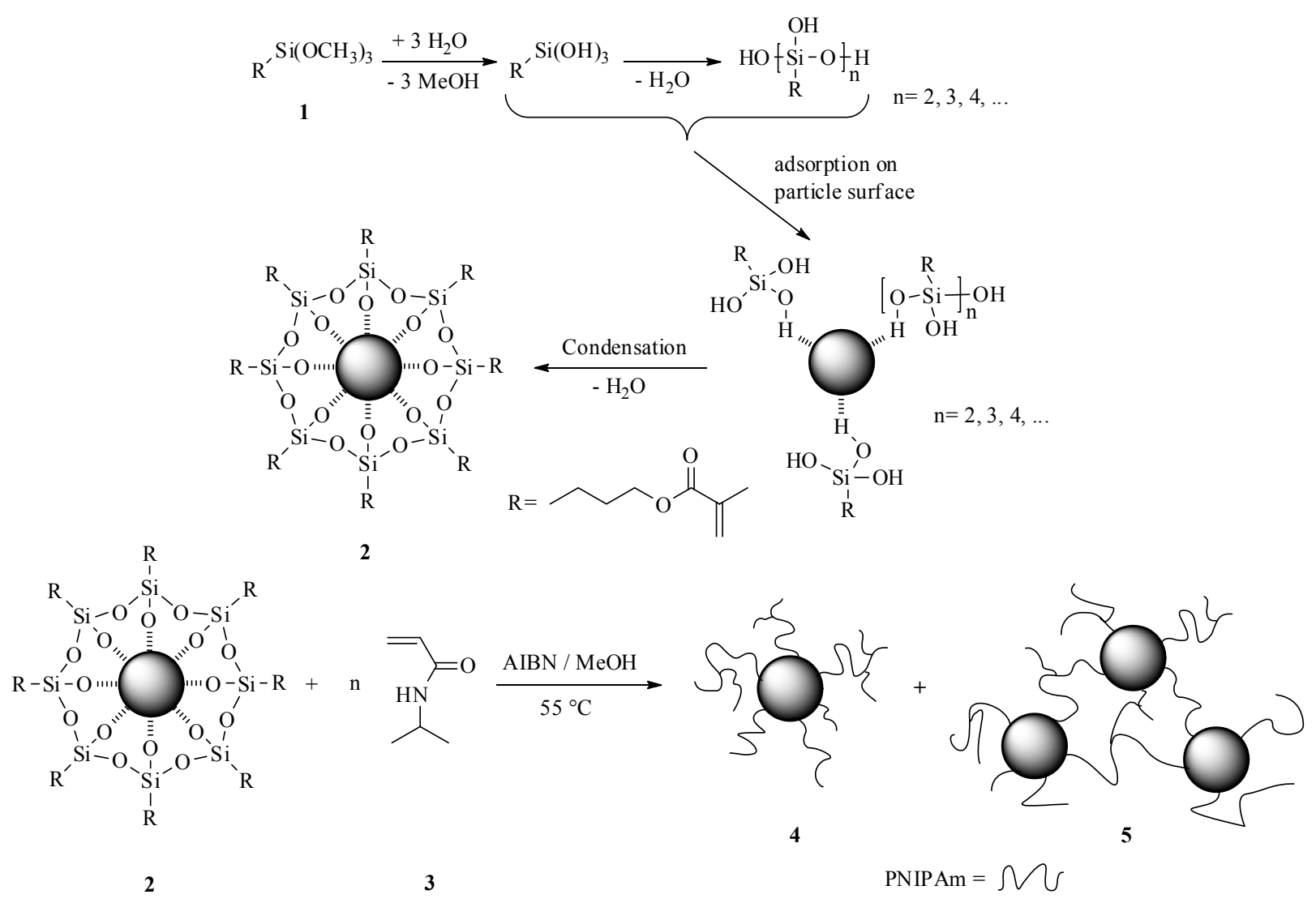

Figure 2. Synthetic pathway used in order to obtain PNIPAm-coated MNPs. 1: MEMO, 2: MNPsMEMO, 3: NIPAm, 4: MNPs-MEMO-PNIPAm and 5: crosslinked MNPs-MEMO-PNIPAm. 
Secondly, the vinyl groups of MNPs-MEMO were used to introduce the PNIPAm shell through a radical homopolymerization of the monomer NIPAm. Different weight ratios of MNPs-MEMO:NIPAm were assayed, observing that in all cases, the conversion of monomers to polymers was complete since no free monomer was detected in the supernatant of each wash. Besides, it was noted that an increase in the monomer concentration leads to products more difficult to process and purify using magnetically-assisted washes. Probably the difficulty to separate the modified MNPs from the aqueous dispersion using the magnet at higher monomer concentrations can be attributed to a "shielding" of the magnetic core by a dense polymer shell.

Moreover, it was observed that the stability of the modified MNPs decreased when the ratio of monomer to nanoparticle increased. At the lowest ratio, the homogenous aqueous dispersion of MNPs-MEMO-PNIPAm is stable for a year, while at the highest ratio the stability of these dispersions reduces to two weeks. In principle, this observation could be explained considering that the use of higher monomer concentrations favours the formation of aggregates of modified MNPs due to secondary interactions (hydrophobic/hydrophilic), entanglement, or crosslinking between the polymer chains bounded to the MNPs. Consequently, a complete characterization of the product MNPs-MEMO-PNIPAm with the lowest monomer ratio (1:6.5) was performed, which will be described in the following sections.

On the other hand, it should be noted that the macroscopic magnetic behaviour of the MNPsMEMO-PNIPAm remains the same, as Figure 3 illustrates. Thus, the purification of this product was assisted by magnetic separation. The product MNPs-MEMO-PNIPAm can be easily separated from a homogeneous aqueous dispersion (Figure 3a) using a Neodymium commercial magnet (Figure 3b), which indicates the magnetic property of the functionalized MNPs.

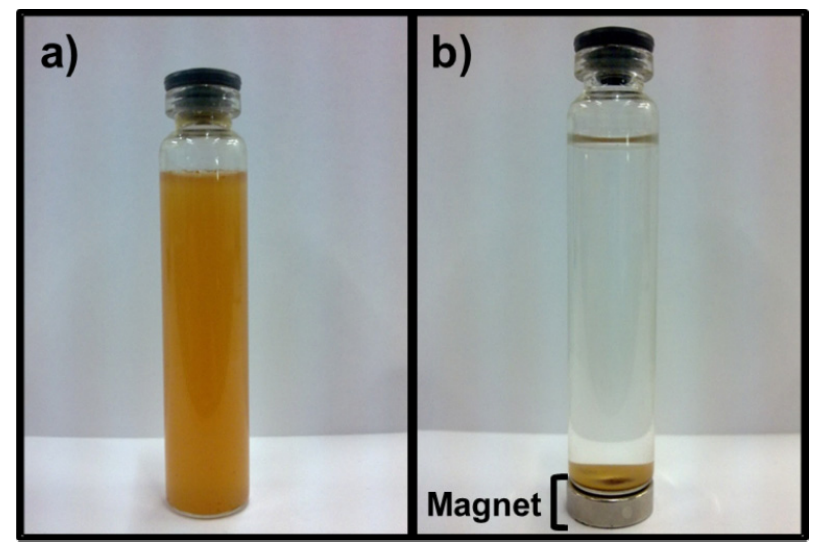

Figure 3. Picture of the MNPs-MEMO-PNIPAm, a) homogeneously dispersed in water and b) caught by a commercial magnet.

\section{Characterization of PNIPAm modified MNPs}

IR studies. The success of each synthetic step was initially followed by IR spectroscopy, as shown in Figure 4. Figure 4a corresponds to MNPs and depicts the characteristic Fe-O 
absorption bands at 560 and $633 \mathrm{~cm}^{-1}$, which are highly consistent with the spectrum of maghemite. ${ }^{13,14}$

The immobilization of MEMO on the MNP surface to achieve MNPs-MEMO (Figure 4b) was confirmed by the presence of the following absorption bands: $2960-2850 \mathrm{~cm}^{-1}$ (aliphatic C$\mathrm{H}$ stretching vibrations), $1639 \mathrm{~cm}^{-1}\left(\mathrm{C}=\mathrm{C}\right.$ stretching), $1710 \mathrm{~cm}^{-1}$ ( $\mathrm{C}=\mathrm{O}$ stretching), $1160 \mathrm{~cm}^{-1}$ (Fe-O-Si bonds between MNPs and MEMO), and $1015 \mathrm{~cm}^{-1}$ (Si-O-Si bonds, attributed to the self-condensation reaction undergone by the silane groups to form a polysiloxane film on the MNPs surface). ${ }^{12,15}$ The band at $3400 \mathrm{~cm}^{-1}$ is attributed to $\mathrm{O}-\mathrm{H}$ stretching of the remnant -OH groups.

Figure $4 \mathrm{c}$ shows the IR spectrum of MNPs-MEMO-PNIPAm, where the following bands should be noted: $3430 \mathrm{~cm}^{-1}$ (N-H stretching of PNIPAm amide), 1645, and $1545 \mathrm{~cm}^{-1}$ (secondary amide $\mathrm{C}=\mathrm{O}$ stretching and $\mathrm{N}-\mathrm{H}$ stretching of the $\mathrm{CONH}$ groups of the NIPAm polymer chains, respectively), 1390 and $1370 \mathrm{~cm}^{-1}$ (C-H bending of isopropyl groups). In addition, the absence of the following absorption bands corresponding to free NIPAm monomers: $1640-1660 \mathrm{~cm}^{-1}$ and $1535-1540 \mathrm{~cm}^{-1}$ the $\left(\mathrm{C}=\mathrm{O}\right.$ stretching (amide I) and N-H bending (amide II), respectively), ${ }^{16}$ and $1630 \mathrm{~cm}^{-1}(\mathrm{C}=\mathrm{C}$ stretching $)$ confirmed that no unreacted monomer remained in the aqueous dispersion after the reaction.

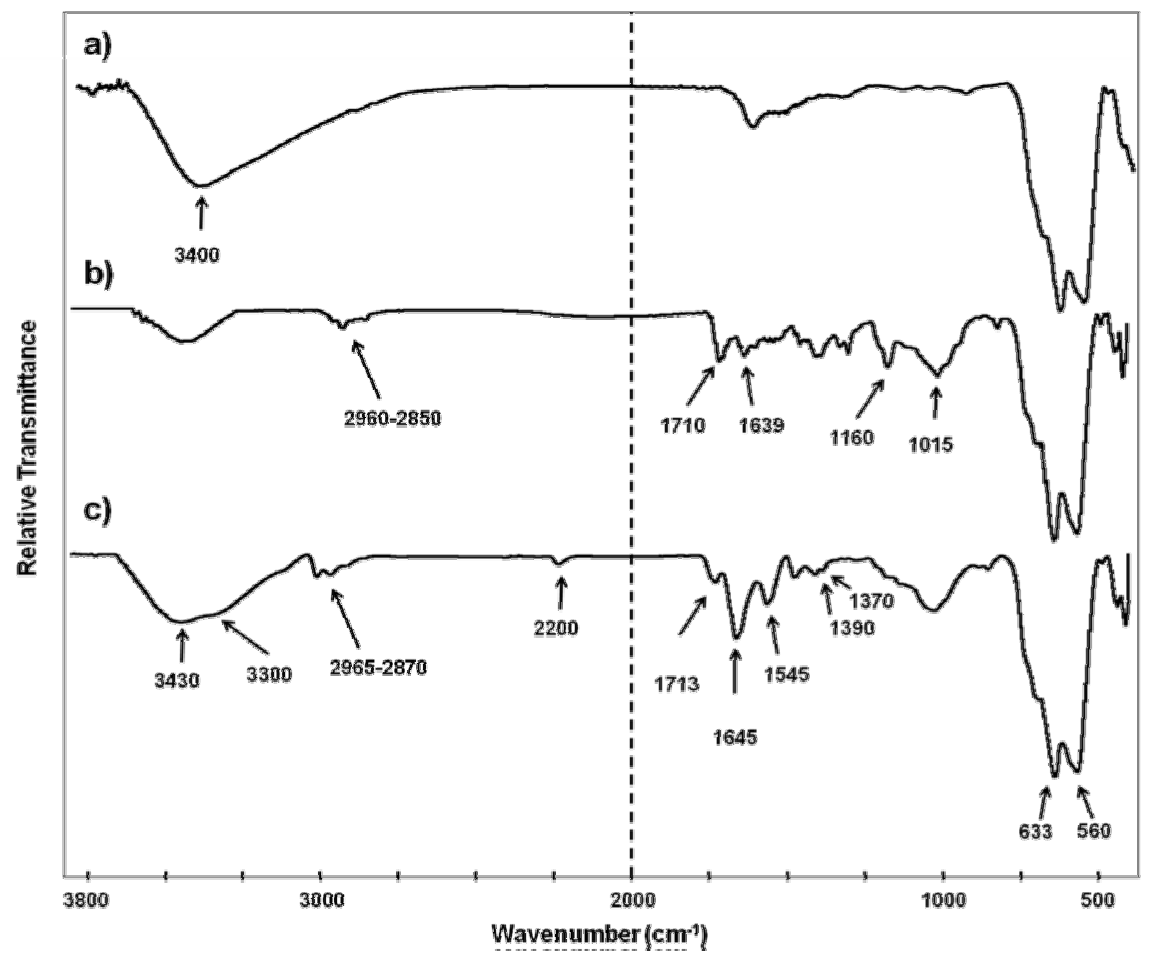

Figure 4. FT-IR spectra of a) MNPs, b) MNPs-MEMO and c) MNPs-MEMO-PNIPAm.

TGA studies. Figure 5 shows the thermograms obtained for the original MNPs and the modified MNPs, in a temperature range of $25-500{ }^{\circ} \mathrm{C}$. MNPs (Figure 5a) show weight loss of about $2 \%$ 
corresponding to water loss. It is well-known that from 25 to $400{ }^{\circ} \mathrm{C}$ the physically adsorbed water is removed, whereas over $400{ }^{\circ} \mathrm{C}$ a chemical dehydration process takes place (Figure 6a). ${ }^{17}$ MNPs-MEMO (Figure 5b) undergoes mass loss in the range of $9 \%$ corresponding to the evaporation of water and the decomposition of MEMO. It has been reported that the thermal degradation of MEMO proceeds as follows: first, there is a cleavage of $\mathrm{C}-\mathrm{O}$ bonds (methacrylic acid loss by McLafferty rearrangement) (Figure 6b) followed by the cleavage of $\mathrm{Si}-\mathrm{C}$ bonds (allylic radical loss). After this degradation, a $\mathrm{SiO}_{2}$ shell remains on the particle surface. ${ }^{17}$

Finally, Figure $5 \mathrm{c}$ shows the percentage of mass loss of MNPs-MEMO-PNIPAm to be about $90 \%$. The weight loss around $400{ }^{\circ} \mathrm{C}$ is associated with the degradation of the $\mathrm{N}$ isopropylacrylamide groups. ${ }^{18,19}$ According to the TGA curves in Figure 5, the calculated mass of PNIPAm was around $79 \%$. This value is considerably higher than that determined by Schmidt ${ }^{12}$ in similar studies, and this difference could be explained considering that, in the present work, a considerably higher monomer ratio was employed, implying a higher organic modification of the MNPs.

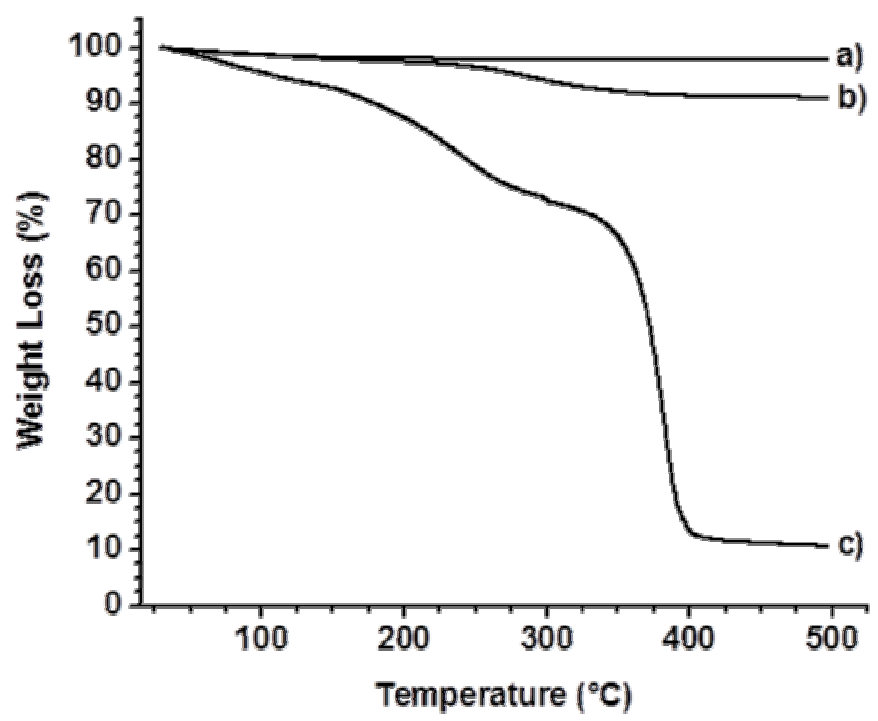

Figure 5. TGA curves of a) MNPs, b) MNPs-MEMO, and c) MNPs-MEMO-PNIPAm.

a)

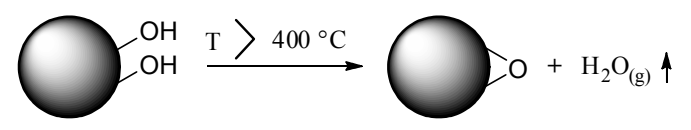

b)

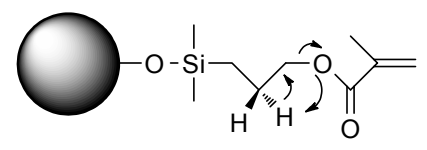

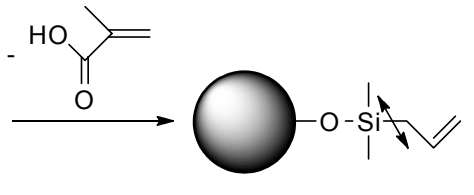

Figure 6. a) chemical dehydration process of MNPs, b) methacrylic acid loss by McLafferty rearrangement in the thermal degradation of MEMO.

\section{Study of the thermo-responsive properties of MNPs-MEMO-PNIPAm}

UV-VIS studies: determination of the lower critical solution temperature (LCST). It is well known that PNIPAm has a very fast response to thermal stimuli; Heskins and Guillet $^{20}$ found that 
heating an aqueous solution of PNIPAm above $32{ }^{\circ} \mathrm{C}$ induces phase separation. Such thermal response involves a transformation from the swollen state under LCST to the shrunk state above LCST, corresponding to the "intermolecular" and "intramolecular" hydrogen bonds, respectively.

In order to understand the thermo-sensitive character of a dispersion of MNPs-MEMOPNIPAm, the change in absorbance at $\lambda=500 \mathrm{~nm}$ as a function of temperature was plotted and analyzed (Figure 7). The LSCT $=32.5^{\circ} \mathrm{C}$ was calculated from the first derivative of the plot, and this value is in very good agreement with a previously reported LCST of $32{ }^{\circ} \mathrm{C}$, determined for PNIPAm. ${ }^{21-26}$

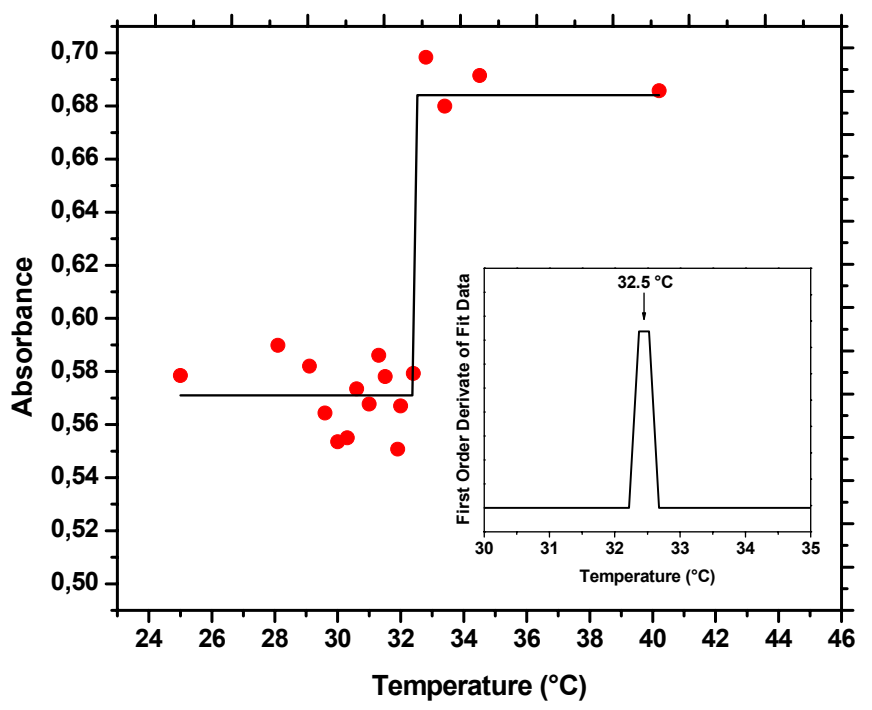

Figure 7. LCST determination of a MNPs-MEMO-PNIPAm dispersion by UV-vis.

DLS studies. In order to further study the thermo-responsive behavior observed by UV-vis studies, DLS measurements were conducted. Figure 8 shows the evolution of the averaged particle size measured of a MNPs-MEMO-PNIPAm aqueous dispersion as a function of temperature, where two regions can be observed. From 25 to $33{ }^{\circ} \mathrm{C}$, a decrease $(\mathrm{ca}$. $19 \%$, from 165 to $135 \mathrm{~nm}$ ) in particle size is observed; from 33 to $50{ }^{\circ} \mathrm{C}$ the particle size increases (from 135 to $160 \mathrm{~nm})$.

It is well known that in pure water, below LCST PNIPAm chains are swollen by uptake of the solvent. Above LCST a phase transition takes place and water is expelled to a certain extent thus a volume phase transition is observed. ${ }^{27}$

Therefore, in the present work the particle size decrease from 25 to $33{ }^{\circ} \mathrm{C}$ is attributed to the deswelling of the PNIPAm chains, according to UV-vis results. From 33 to $50{ }^{\circ} \mathrm{C}$, particle size increase is probably related to an aggregation process undergone by the modified MNPs, where solute-isoPr group interactions are energetically more favorable for isoPr groups in a polymer rich environment compared to isoPr groups in water. ${ }^{28}$ This effect would be similar to the 
aggregation process reported for other hybrid materials with a different polymer where a thermally triggered hydrophilic-to-hydrophobic phase transition occurred. ${ }^{29}$

It is worth mentioning that the particle sizes here reported are considerably bigger than those previously reported by others authors. Probably, this difference is due to the presence of some aggregates of modified MNPs even at the lowest temperature, as was suggested above. With the aim of corroborate this assumption, TEM measurements were carried out.

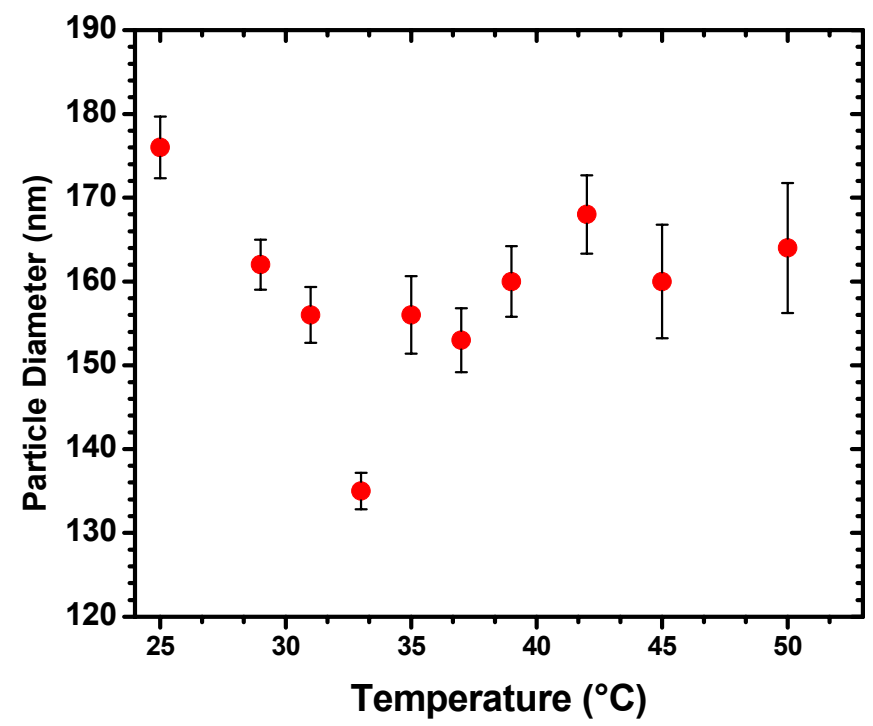

Figure 8. DLS measurement of the particle size of aqueous MNPs-MEMO-PNIPAm dispersion with varying temperature.

\section{TEM study of temperature-dependent aggregation}

As mentioned above, in order to have more conclusive evidence about the thermo-sensitive properties of MNPs-MEMO-PNIPAm dispersions and with the aim to support the results obtained by DLS, TEM images of the samples at different temperatures were taken after each experiment.

Figure 9 shows TEM images of MNPs-MEMO-PNIPAm at two different magnifications and at five different temperatures. The images show clearly the presence of aggregates at all temperatures, according to DLS results. However, some differences can be pointed out. Below LCST $=33{ }^{\circ} \mathrm{C}$ (determined by DLS) there are dispersed nanoparticles together with aggregates (Figure 9a-d). Above LCST, the aggregation is highly favored and large aggregates are observed while there are only a few dispersed nanoparticles (Figure 9e-j). It is worth mentioning that TEM studies are conducted in the solid state and probably the drying of the sample may affect the described process.

Another important feature is that the volume transition of the PNIPAm shell with temperature can be noticed. Below LCST, a thick polymer shell located in the surface of the nanoparticles is easily observed (Figure 9d) while above LCST this shell becomes very thin and 
almost unobservable in TEM micrographs (Figure 9j) due to the polymer collapse resulting from its thermo-sensitive character.
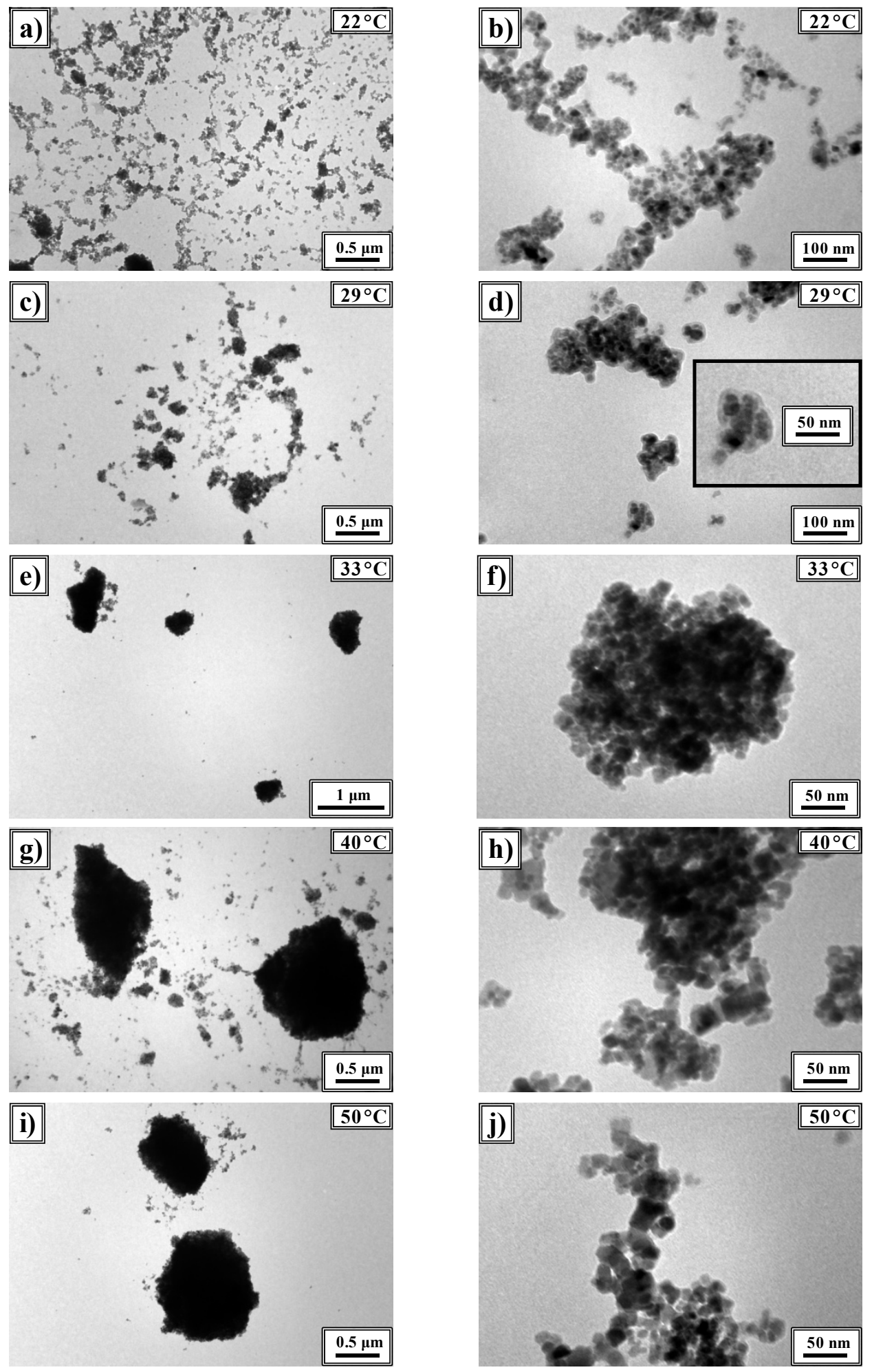

Figure 9. TEM micrographs of MNPs-MEMO-PNIPAm at different temperatures and magnifications. 
Although DLS and TEM measurements are conducted in different phases (in solution and in the solid state, respectively), they are both qualitatively in good agreement with each other and offer complementary information related to the thermo-responsive behavior of the modified MNPs and the presence of aggregates in the present study. In this regard, an aspect which remains to be figured out is the origin of such aggregates. We consider that the aggregates observed at lower temperatures might be originated during the second step of the MNPs-MEMOPNIPAm synthesis due to crosslinking reactions between the growing polymer chains of the modified MNPs. Thus, the mentioned polymerization reaction gives rise to isolated polymermodified MNPs (product 4, Figure 2) and some aggregated modified MNPs (product 5, Fig. 2).

However, the high stability of the aqueous MNPs-MEMO-PNIPAm dispersions (with a monomer ratio of 1:6.5) should be pointed out since no precipitate was detected for several months.

\section{Conclusions}

In summary, we were able to synthesize modified maghemite nanoparticles via a "grafting from" methodology. The experimental procedure involved two synthetic steps: first, the covalent binding of a silane coupling agent (MEMO) to the surface of the MNPs; and secondly, the growing of PNIPAm chains by surface free radical polymerization achieving PINPAm-grafted MNPs. This approach is a simple and low cost methodology which can be used to obtain MNPs modified with an organic shell.

Aqueous MNPs-MEMO-PNIPAm dispersions were stable for a long period of time and showed thermo-sensitive properties with a solution LCST of $33{ }^{\circ} \mathrm{C}$. Besides, they maintain the macroscopic magnetic properties, characteristic of maghemite nanoparticles. Magnetic and thermo-sensitive properties of the MNPs-MEMO-PNIPAm are critical for their potential applications in biomedical and bioengineering fields.

Current studies to better control the polymerization step are in course, aiming to obtain thermoresponsive MNPs useful in drug delivery systems or hyperthermia.

\section{Experimental Section}

Materials and equipment. 3-methacriloxypropyltrimethoxysilane (MEMO) was purchased from Sigma-Aldrich. Maghemite $\left(\gamma-\mathrm{Fe}_{2} \mathrm{O}_{3}\right)$ iron oxide magnetic nanoparticles (MNPs), with a nominal size of $(9.5 \pm 1.1) \mathrm{nm}$ as determined by TEM, were kindly provided by Prof. Iñaki Mondragón (Universidad Del País Vasco, Spain) and were used as received. Organic solvents such as methanol, toluene and $n$-hexane were analytical grade and obtained from various sources. Water was purified with a Millipore Milli-Q system. Toluene and methanol were dried 
over $4 \AA$ molecular sieves. The rest of the chemicals were used as received. All solutions were prepared immediately prior to their use.

Infrared spectroscopy (FT-IR) was carried out on a Nicolet-5SXC, using $\mathrm{KBr}$ or $\mathrm{AgBr}$ pellets; thermogravimetric analysis (TGA) studies were performed on a 2950TGAHR thermogravimetric analyzer (TA Instruments) between room temperature and $500{ }^{\circ} \mathrm{C}$, and at a heating rate of $10^{\circ} \mathrm{C} / \mathrm{min}$, under nitrogen flow. Dynamic Light Scattering (DLS) experiments were carried out using a Delsa NanoC Particle Analyzer Beckman Coulter. Powder X-ray diffraction (PXRD) pattern was measured with a conventional PANalytical XPERT Pro diffractometer, using $\mathrm{Cu}-\mathrm{Ka}$ radiation obtained at $40 \mathrm{kV}$ and $40 \mathrm{~mA}$. The pattern was measured between $10^{\circ}$ and $100^{\circ}(2 \theta)$, with a step of $0.02^{\circ}(2 \theta)$ and counting time of $5 \mathrm{~s} / \mathrm{step}$. UV-Visible turbidimetry experiments were measured on a diode-array Shimadzu Multispec 1501 spectrophotometer coupled with a temperature controller. The cloud point was observed at $500 \mathrm{~nm}$ light beam through a $1 \mathrm{~cm}$ sample cell against Milli-Q water as reference. The spectra were recorded between 400-600 nm at controlled temperature using a $1 \mathrm{~cm}$ optic path quartz cuvette. TEM images were obtained with a JEM 1200EXII-JEOL electron microscope operating at $80 \mathrm{kV}$.

\section{Synthesis of MNPs-MEMO}

The functionalization of MNPs with MEMO was achieved following procedures similar to those previously reported. ${ }^{12,30,31}$ Five different molar ratio of MEMO:MNPs were used (2.5:1; 3.5:1; $5: 1 ; 6.5: 1)$. A typical procedure for the synthesis of MNPs-MEMO using a ratio of 5:1 is described as follows. In a dry three-necked flask equipped with a nitrogen inlet, MNPs (200 mg, $1.25 \mathrm{mmol})$ were dispersed in dry toluene $(15 \mathrm{~mL})$. Then, MEMO $(1.5 \mathrm{~mL}, 6.25 \mathrm{mmol})$ was added using a syringe and under sonication. Note that an excess of MEMO was used. The dispersion was sonicated for $3 \mathrm{~h}$ at room temperature and a stechiometric quantity of water (related to MEMO) was added to promote the hydrolysis of MEMO and its absorption onto the MNP surface. Condensation of the siloxane groups was promoted by azeotropic distillation under reduced pressure in which the water is removed. After distillation, the MNPs were subsequently washed six times with $n$-hexane and centrifuged in order to remove the remaining MEMO. The modified MNPs were dried under vacuum at $45{ }^{\circ} \mathrm{C}$ under vacuum and characterized.

\section{Synthesis of MNPs-MEMO-PNIPAm}

PNIPAm chains were grown from the MNPs-MEMO surface through a radical polymerization. Three different weight ratio of NIPAm:MNPs-MEMO were used (6.5:1, 12:1 and 20:1). A typical procedure for the synthesis of MNPs-MEMO-PNIPAm using a ratio of 6.4:1 is described as follows. In a dry three-necked flask equipped with a nitrogen inlet, MNPs-MEMO (50 mg), NIPAm (0.319 g, $2.82 \mathrm{mmol})$, and AIBN ( $5 \mathrm{mg}, 0.03 \mathrm{mmol})$ were dispersed in dry methanol (5 $\mathrm{mL}$ ) at room temperature under vigorous magnetic stirring for $30 \mathrm{~min}$. Then, a reflux system was coupled; the temperature was raised to $55^{\circ} \mathrm{C}$ and kept for $4 \mathrm{~h}$. Next, methanol was distilled off under reduced pressure. The resulting nanoparticles were washed with $n$-hexane (recovering 
them by magnetic separation) several times until no free monomer or polymer chains unbounded to the MNP surface were detected in the supernatant by FT-IR. The product was dried at $30^{\circ} \mathrm{C}$ under vacuum and characterized.

\section{UV-VIS studies: Determination of the LCST of MNPs-MEMO-PNIPAm} A dispersion of MNPs-MEMO-PNIPAm with a concentration of $2.5 \mathrm{mg} / \mathrm{mL}$ was placed in a 1 $\mathrm{cm}$ optical path liquid cuvette, then sonicated for $10 \mathrm{~min}$ at controlled temperature and immediately measured. The experiments were developed at a temperature range of $25-40{ }^{\circ} \mathrm{C}$. Afterwards, the variation in the absorbance at $\lambda=500 \mathrm{~nm}$ in function of the temperature of the dispersion was plotted and analyzed. The data were fitted with a Boltzmann Sigmoidal Function. The LSCT was estimated from the first derivative of the plot.

DLS measurements. A dispersion of MNPs-MEMO-PNIPAm was prepared by dispersing 10 $\mathrm{mg}$ of product in $5 \mathrm{~mL}$ of water. The dispersion was sonicated for $30 \mathrm{~min}$ at controlled temperature, transferred to a $1 \mathrm{~cm}$ optical path glass cuvette and introduced in the analyzer. The sample was thermally stabilized for 5 min before each measurement. In every experiment only a single population of specimens was found.

TEM studies: evaluation of the temperature-dependent aggregation process. A dispersion of MNPs-MEMO-PNIPAm was prepared by dispersing $10 \mathrm{mg}$ of product in $5 \mathrm{~mL}$ of water. The dispersion was sonicated for $30 \mathrm{~min}$ at controlled temperature. Then, an aliquot was diluted (approximately 7 times) to a proper concentration in a thermostatic bath. Samples for TEM were prepared by drop-casting one drop of the as-prepared dispersion onto a standard carbon-coated Formvar copper grid (200-mesh) and keeping it at $25^{\circ} \mathrm{C}$ until complete solvent evaporation. For each sample, at least four typical regions were scanned. The images were analyzed using the ImageJ software.

\section{Acknowledgements}

The authors gratefully acknowledge financial support from CONICET, ANPCyT, and SECYT of Universidad Nacional de Córdoba. A.L.C and J.I.P. wish to thank CONICET for the fellowships provided. A.L.C. thanks Lic. Julio Cuggino for helpful discussion.

\section{References}

1. Banerjee, S. S.; Chen, D. H. Int. J. Appl. Ceram. Technol. 2010, 7, 111.

2. Kim, D. J.; Heo, J.; Kim, K.; Choi, I. Macromol. Rapid Commun. 2003, 24, 517.

3. López-León, T; Ortega-Vinuesa, J. L.; Bastos-González, D.; Elaïssari, A. J. Phys. Chem. B 2006, $110,4629$. 
4. Fernández-Nieves, A.; Fernández-Barbero, A.; Vincent, B.; De las Nieves, F. J. J. Chem. Phys. 2003, 119, 10383.

5. Vihola, H.; Laukkanen, A.; Valtola, L.; Tenhu, H.; Hirvoenen, J. Biomaterials 2005, 26, 3055 .

6. Pankhurst, Q. A.; Connolly, J.; Jones, S. K.; Dobson, J. J. Phys. D. Appl. Phys. 2003, 36, 167.

7. Dobson, J. Drug. Dev. Res. 2006, 67, 55.

8. Georgelin, T.; Maurice, V.; Malezieux, B.; Siaugue, J. M.; Cabuil, V. J. Nanopart. Res. 2010, 12, 675 .

9. Omer, M.; Haider, S.; Park, S. Polymer 2011, 52, 91.

10. Zhu, Y.; Kaskel, S.; Ikoma, T.; Hanagata, N. Micropor. Mesopor. Mat. 2009, 123, 107.

11. Wang, S.; Zhou, Y.; Sun, W. Materials Sci. Eng. C 2009, 29, 1200.

12. Frickel, N.; Messing, R.; Gelbrich, T.; Schmidt, A. M. Langmuir 2010, 26, 2839

13. Darezereshki, E. Mater. Lett. 2010, 64, 1471.

14. Asuha, S.; Zhao, S.; Wu, H. Y.; Song, L.; Tegus, O. J. Alloy. Compd. 2009, 472, 23

15. Flesch, C.; Delaite, C.; Dumas, P.; Bourgeat-Lami, E.; Duguet, E. J. Polym. Sci. Part A: Polym. Chem. 2004, 42, 6011.

16. Ji, X.; Shao, R.; Elliott, A. M.; Stafford, R. J.; Esparza-Coss, E.; Bankson, J. A.; Liang, G.; Luo, Z.-P.; Park, K.; Markert, J. T.; Li, C. J. Phys. Chem. C 2007, 111, 6245.

17. Abboud, M.; Turner, M.; Duguet, E.; Fontanille, M. J. Mater. Chem. 1997, 7, 1527.

18. Sousa, R. G.; Magalhaes, W. F.; Freitas, R. F. S. Polym. Degrad. Stab. 1998, 61, 275.

19. Zhang, H.; Zhong, H.; Zhang, L.; Chen, S.; Zhao, Y.; Zhu, Y. Carbohyd. Polym. 2009, 77, 785.

20. Heskins, M.; Guillet, J. E. J. Macromol. Sci., Part A: Pure Appl. Chem. 1968, 2, 1441.

21. Hirokawa, Y.; Tanaka, T. J. Chem. Phys. 1984, 81, 6379.

22. Hoffman, A. S. MRS Bull. 1991, 16, 42.

23. Takei, Y. G.; Aoki, T.; Sanui, K.; Ogata, N.; Sakurai, Y.; Okano, T. Macromolecules 1994, $27,6163$.

24. Liang, L.; Feng, X.; Liu, J.; Rieke, P. C.; Fryxell, G. E. Macromolecules 1998, 31, 7845.

25. Zhang, X. Z.; Zhuo, R. X. Langmuir 2001, 17, 12.

26. Ni, C.; Zhu, X. Eur. Polym. J. 2004, 40, 1075.

27. Lu, Y.; Ballauff, M. Prog. Polym. Sci. doi:10.1016/j.progpolymsci.2010.12.003.

28. Pelton, R. J. Colloid Interf. Sci. 2010, 348, 673.

29. Nath, N.; Chilkoti, A. J. Am. Chem. Soc. 2001, 123, 8197.

30. Ishida, Y.; Jikei, M.; Kakimoto, M. Macromolecules 2000, 33, 3202.

31. Marutani, E.; Yamamoto, S.; Ninjbadgar, T.; Tsujii, Y.; Fukuda, T.; Takano, M. Polymer 2004, 45, 2231.

32. Cullity, B. D. Elements of X-ray Diffraction, Addison- Wesley, Reading, MA, 1967, p. 99. 